\title{
Bolus clearance in esophagogastric junction outflow obstruction is associated with strength of peristalsis
}

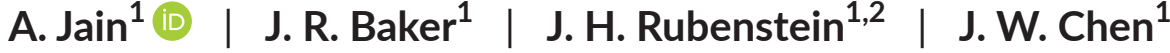

${ }^{1}$ Division of Gastroenterology, University of Michigan Health System, Ann Arbor, Michigan, USA

${ }^{2}$ Veterans Affairs Center for Clinical Management Research, Ann Arbor, Michigan, USA

\section{Correspondence}

Joan W. Chen, MD, MS, 3912 Taubman

Center, 1500 E Medical Center Dr., SPC 5362, Ann Arbor, MI 48109, USA.

Email: chenjoan@med.umich.edu

Funding information

None.

\begin{abstract}
Background: A manometric diagnosis of esophagogastric junction outflow obstruction (EGJOO) without a mechanical cause creates a therapeutic conundrum. The aim of this study was to assess esophageal bolus clearance in EGJOO and assess manometric factors associated with clearance in EGJOO.

Methods: Bolus clearance was assessed using line-tracing method and contour method to determine Complete Bolus Transit (CBT) and Functional Clearance (FC), respectively, on combined High-Resolution Impedance Manometry (HRIM). HRIM studies of EGJOO patients, as well as a sample of achalasia types I-III and asymptomatic controls, were retrospectively analyzed. In EGJOO, associations between Integrated Relaxation Pressure (IRP) or Distal Contractile Integral (DCl) and clearance were assessed using receiver-operating-characteristic $(\mathrm{ROC})$ curves.
\end{abstract}

Key Results: Seventy-five EGJOO, 28 achalasia, and 11 normal subjects were included. Agreement between CBT and FC was good (Kappa=0.75). CBT across swallows in each group was as follows: type I achalasia: $14 \%$, type II achalasia: $8 \%$, type III achalasia: 61\%, EGJOO: $86 \%$, and normal: $98 \%$ (p values $.023, .006$, and $<.0001$ for EGJOO vs normals, type III achalasia, and all achalasia, respectively). In idiopathic EGJOO, CBT $\geq 60 \%$ of swallows was seen in $96.4 \%$ of patients when mean $\mathrm{DCl}>610 \mathrm{mmHg}-\mathrm{s}-\mathrm{cm}$ (accuracy $87.7 \%, P=.004)$. Complete Bolus Transit( $C B T$ ) across individual swallows was $97.8 \%$ when $\mathrm{DCl}>884 \mathrm{mmHg}-\mathrm{s}-\mathrm{cm}$ (accuracy $81.9 \%, P<.0001$ ). IRP was poorly associated with bolus clearance.

Conclusions \& Inferences: Bolus clearance in EGJOO is impaired compared to normal, but not as severely as in achalasia. In idiopathic EGJOO, weak peristalsis is associated with poor bolus clearance. Bolus transit appears to be unimpaired when $\mathrm{DCl}>900 \mathrm{mmHg}-\mathrm{s}-\mathrm{cm}$.

KEYWORDS

esophagus, High-resolution manometry, impedance, motility

Abbreviations: AUC, Area-under-the-curve; CBT, Complete Bolus Transit; DCI, Distal Contractile Integral; EGJ, Esophagogastric junction; EGJOO, Esophagogastric junction outflow obstruction; EPT, Esophageal pressure topography; FC, Functional Clearance; HRIM, High-Resolution Impedance Manometry; HRM, High-resolution manometry; IDQ, Impaction Dysphagia Questionnaire; IQR, Interquartile Range; IRB, Institutional review board; IRP, Integrated Relaxation Pressure; PRO, Patient Reported Outcomes; RNYGB, Roux-en-Y Gastric Bypass; ROC, Receiver Operating Characteristic; UGI, Upper Gastrointestinal. 


\section{1 | INTRODUCTION}

The transition from conventional manometry to high-resolution manometry (HRM) with esophageal pressure topography (EPT) in recent decades has enhanced the detection and interpretation of esophageal motor disorders. Novel metrics for EPT and a classification system have been devised to characterize esophageal function. In the second and third iterations of the Chicago Classification, characterization of the esophagogastric junction (EGJ) has been proposed as the first step in classifying esophageal motility disorders. ${ }^{1}$ This has led to the new manometric diagnosis of esophagogastric junction outflow obstruction (EGJOO). In the latest iteration of the Chicago Classification version 3.0, EGJOO was defined as a median IRP of greater than $15 \mathrm{~mm} \mathrm{Hg}$ along with preserved peristalsis in the esophageal body. ${ }^{2,3}$

The etiology and clinical relevance of EGJOO are not fully understood. Anatomic obstructions such as prior fundoplication or esophageal stenosis offer straightforward explanations. Other structural abnormalities such as hiatal hernia and eosinophilic esophagitis are plausible explanations. However, significant portions of manometrically identified EGJOO have no associated mechanical obstruction and are termed idiopathic EGJOO. ${ }^{4-7}$ The clinical presentation of idiopathic EGJOO appears to be heterogeneous. Dysphagia is the most common symptom, affecting $40 \%$ in larger cohorts, followed by chest pain, regurgitation, and heartburn. Notably, a portion of these patients seems to experience spontaneous resolution of symptoms. ${ }^{7}$ The pathophysiology of idiopathic EGJOO is unclear; however, there has been speculation that EGJOO may be a precursor to or a variant of achalasia. This theory is supported by case series of idiopathic EGJOO progressing to achalasia, and by anecdotal response to therapy delivered to the EGJ in some EGJOO patients. ${ }^{4,5}$ There has been great interest in phenotyping EGJOO to determine its functional significance.

Manometry with intraluminal impedance offers an opportunity to better characterize the functional significance of idiopathic EGJOO. Complete Bolus Transit (CBT) has been defined on conventional line-tracing impedance manometry as bolus entry at the proximal most impedance recording site and subsequent exit at all 3 distal recording sites, as indicated by an appropriate drop and return to baseline impedance. CBT in $\geq 8$ of 10 liquid swallows has been seen in the majority of normal individuals. ${ }^{8,9}$ Impaired bolus transit has been shown to correlate with erosive esophagitis in patients with gastro-esophageal reflux disease, dysphagia in patients with large peristaltic breaks, and column height on timed barium esophagram in patients with achalasia. ${ }^{10-12}$ It has also been shown that bolus transit on impedance correlates well with that seen on the gold standard videofluoroscopy. ${ }^{11}$

The aim of our study was to assess bolus transit in idiopathic EGJOO using HRIM and compare this with that seen in achalasia subtypes and normal subjects. We also sought to determine whether phenotyping EGJOO patients based on manometric parameters, Integrated Relaxation Pressure (IRP), and Distal Contractile Integral $(\mathrm{DCl})$, would have functional ramifications on clearance. A sub-aim of

\section{Key Points}

- Esophagogastric junction outflow obstruction (EGJOO) is a manometric diagnosis frequently with unclear clinical significance. We examined bolus clearance and the correlation between clearance and manometric parameters in patients with idiopathic EGJOO.

- Bolus clearance is impaired in EGJOO, but not as severely as in achalasia.

- Weak peristalsis is associated with poor bolus clearance in idiopathic EGJOO. Bolus transit is typically unimpaired when $\mathrm{DCl}$ is greater than $900 \mathrm{mmHg}-\mathrm{s}-\mathrm{cm}$.

the study was to assess the reliability of the colored contour method of assessing bolus transit on HRIM in comparison to the more objective line-tracing method.

\section{2 | MATERIALS AND METHODS}

\section{1 | Study design}

This was a retrospective cohort study. Consecutive HRIM procedures performed in adult patients (older than 18 years of age) in the Gastrointestinal Physiology Lab at the University of Michigan between July 2013 and December 2015 were screened for the diagnosis of EGJOO. HRIM tracings for all patients were reviewed to confirm an accurate diagnosis per the latest Chicago Classification. Patients were characterized as either structural EGJOO if they had a history of fundoplication, or idiopathic EGJOO if there was no known cause of obstruction at the EGJ. Randomly selected patients with diagnoses of achalasia types I-III and patients without esophageal symptoms with normal manometry were also included for comparison. Study protocol was approved by the Institutional Review Board (IRB) at the University of Michigan. All authors had access to the study data and reviewed and approved the final manuscript.

\subsection{Review of medical record}

The electronic medical records of included subjects were reviewed. This included demographic information, medical and surgical history, medications, diagnostic testing such as endoscopy and barium esophagram, weights, and esophageal symptoms including patient reported outcome (PRO) questionnaires.

\section{3 | Manometry and intraluminal impedance measurement}

Patients underwent manometry using a combined High-resolution Manometry and intraluminal impedance (HRIM) system. This is a solidstate assembly with 36 levels of pressure sensors and 12 impedancesensing segments (Medtronics). The response characteristics of this device, calibration procedure, and poststudy thermal correction 
algorithm have been described in detail previously. ${ }^{13}$ Briefly, the HRIM assembly was passed transnasally and positioned to record from the hypopharynx to the stomach with about five intragastric sensors. The manometric protocol included a 5-minute period for acclimatization and to assess basal sphincter pressure followed by ten $5 \mathrm{~mL}$ swallows of $0.45 \%$ saline in the supine position. In studies performed after December of 2013, five additional $5 \mathrm{~mL}$ swallows were also performed in the upright position. The combined pressureimpedance data were manually analyzed using the ManoView ESO v.3.0.1 software (Medtronic Inc, Shoreview, MN, USA). The color isobaric contour plots were overlaid with impedance line tracings by first selecting the "enabling impedance trace on contour" option. After thermal compensation, the HRM studies were analyzed in accordance with the Chicago Classification v3.0. After confirming the diagnosis of EGJOO, data for individual swallows were abstracted by documenting the values for each parameter: distance between LES-crural diaphragm, Integrated Relaxation Pressure (IRP), Distal Contractile Integral (DCI), Distal Latency (DL), and presence of large peristaltic breaks. After this, the display mode button was changed to impedance tracing (colored contour and lines) on contour. Bolus transit was assessed via line-tracing and contour methods.

\section{4 | Bolus Clearance}

\subsection{1 | Complete bolus transit}

Bolus transit was assessed for each swallow using impedance line tracings. Bolus entry was defined as a $>50 \%$ drop in impedance level from baseline at the proximal recording site, and complete bolus transit (CBT) was defined as a $>50 \%$ drop from baseline followed by an increase of at least $50 \%$ towards the original baseline at the three distal impedance recording sites after bolus entry. ${ }^{8}$ Each of the 10 wet swallows, and when available the 5 wet upright swallows, was scored as either complete or incomplete bolus clearance. In instances where the impedance baseline before each swallow was low indicating retained bolus preswallow, impedance baseline during the "landmarkID" measurement period was used as the reference impedance level to assess bolus transit. Examples of complete and incomplete bolus transit are shown in Figure 1.

\subsection{2 | Functional clearance}

Bolus clearance was also assessed via contour method. Impedance data were displayed as a monochrome color gradient overlaying the pressure topography by changing the contour mode. The color contrast, manually adjustable by changing the level of impedance indication in kohm, was adjusted so that the contour showed an emptying esophagus before initiation of the $0.45 \%$ saline swallow sequence, and the liquid ingested with each swallow was just visible. Bolus clearance was defined by the absence of bolus in the distal esophagus (distal 3 leads) after each swallow. Incomplete functional clearance (FC) was defined as evidence of residual fluid bolus in the distal esophagus.

Determination of CBT and FC was performed by a single reader (JC) for the primary data analysis. A second reader (AJ) blindly reviewed $25 \%$ of randomly selected HRIM studies for comparison.

\section{5 | Statistical methods}

CBT and FC were determined for each of 10 supine swallows and 5 upright swallows when available. Mean CBT and FC frequencies per patient were calculated for achalasia subtypes, EGJOO and normal across supine swallows. Median CBT and FC frequencies per patient were compared between above groups. Within EGJOO, CBT and FC frequencies and $\mathrm{DCl}$ and IRP were compared between idiopathic and structural groups. Kappa statistic was calculated between CBT and FC across supine swallows in all groups, and separately across upright swallows in EGJOO group. Kappa was also calculated for CBT and FC across swallows between both readers in the $25 \%$ randomly selected EGJOO HRIM studies. Within the idiopathic EGJOO group,
(A)

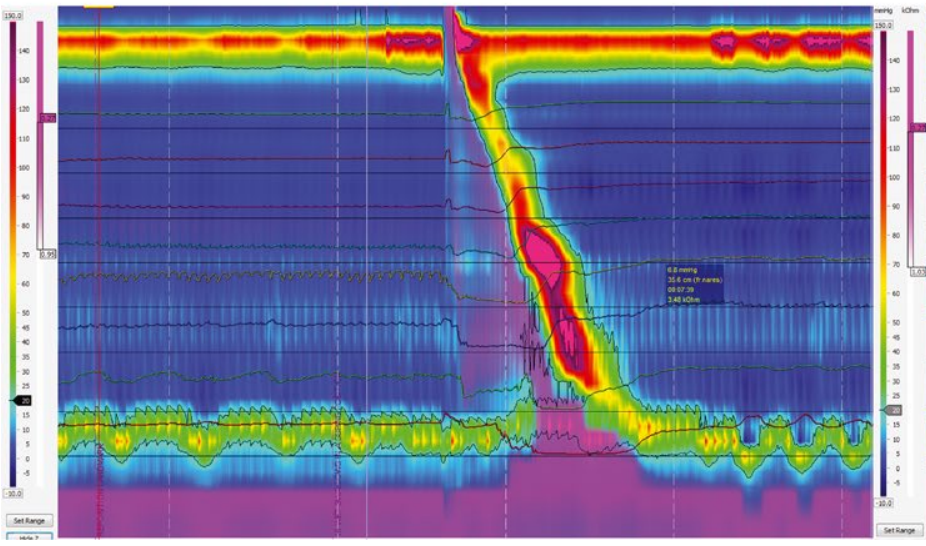

(B)

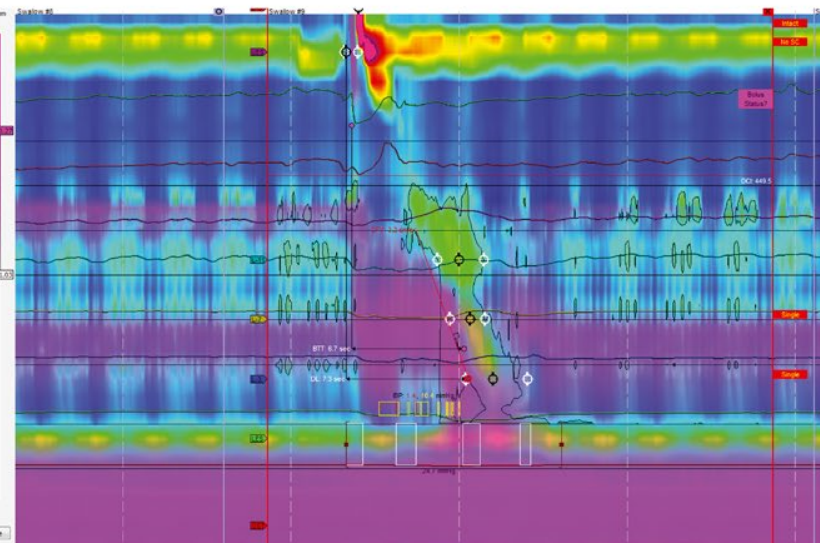

FIGURE 1 HRIM in an EGJOO subject with complete bolus clearance-(A) Pressure topography showing normal esophageal peristalsis and impaired LES relaxation, consistent with EGJ outflow obstruction. (B) Using the trace-on-contour mode and overlaying impedance data, complete bolus clearance is seen. Impedance data in komh can be found using the smart mouse hovering over the contour at specific catheter depths. 
receiver operating characteristic $(\mathrm{ROC})$ curves were constructed for CBT based on $\mathrm{DCl}$ (per swallow or mean of supine swallows) and IRP (per swallow or median of supine swallows) for three outcomes-CBT $\geq 60 \%$ per patient, $\mathrm{CBT} \geq 80 \%$ per patient, and CBT across individual supine swallows. An ROC curve for clearance as defined as CBT on all five upright swallows based on mean $\mathrm{DCl}$ was also constructed. Cutoff values for DCl were selected in each mode using Youden's index. Parametric variables were analyzed via unpaired $t$ test with Welch's correction. Non-parametric variables were analyzed via Mann-Whitney test. Categorical variables were analyzed using Fisher's exact test. A p value of $\leq .05$ was considered statistically significant for all analyses. On ROC curves, an area under the curve (AUC) less than 0.70 was deemed insufficient for threshold analysis. All statistical analyses were performed using GraphPad Prism v7.0, La Jolla California, USA.

\section{3 | RESULTS}

\section{1 | Sample Description}

Seventy-five adult patients with a manometric diagnosis of EGJOO were included in this analysis. Five type I achalasia, 5 type II, 18 type III, and 11 normal HRIM studies were included for comparison. Ten of the EGJOO patients had a structural cause due to prior fundoplication. The remaining 65 were classified as idiopathic EGJOO. Fiftyseven (88\%) of these had HRM protocol including upright swallows. Demographics and relevant clinical data for EGJOO patients are displayed in Table 1. Dysphagia was the most common symptom, reported by $68 \%$ of patients. Four patients were asymptomatic with testing done as part of lung transplant evaluation. Ten patients had a hiatal hernia diagnosed on HRM, and five patients had gastric surgery other than a Nissen fundoplication. Twenty-eight percent of patients had a prescription for narcotics at the time of HRIM. Dysmotility was the most common abnormality on esophagram, noted in $43.8 \%$ of patients. Findings on upper endoscopy were rare, and retained fluid was not seen in any patients. Manometric parameters and clearance for swallows in idiopathic EGJOO and structural EGJOO patients are displayed in Table 2.

\section{2 | Bolus transit}

Mean CBT and FC per patient in all groups are shown in Figure 2. Kappa coefficient for CBT and FC across all supine swallows was good at 0.75 . Esophagogastric junction outflow obstruction patients had lower CBT than healthy controls ( $86 \%$ vs $98 \%, P=.023$ ) but higher than type III achalasia patients $(61 \%, P=.006)$ or achalasia patients as a whole (43\%, $P<.0001)$. Similarly, FC frequency in EGJOO (79\%) was lower than in controls (98\%, $P=.013)$, but higher than in type III achalasia (54\%, $P=.035)$ and achalasia as a whole $(35 \%$, $P<.0001)$.

Agreement in CBT and FC in 19 (25\%) randomly selected EGJOO HRIM studies with a second reader was excellent (kappa=0.96 and 0.86 , respectively).
TABLE 1 Clinical data in EGJOO $(\mathrm{N}=75)$

\begin{tabular}{|lc|}
\hline Male & 21 \\
\hline Female & 54 \\
\hline Age (years) (mean $[\mathrm{SD}])$ & $57.81(13.5)$ \\
\hline Body Mass Index $\left(\mathrm{kg} / \mathrm{m}^{2}\right)$ (mean $\left.[\mathrm{SD}]\right)$ & $28.58(8.55)$ \\
\hline Symptoms $(\%)$ & \\
\hline Dysphagia & $51(68.0)$ \\
\hline Heartburn and/or regurgitation & $24(32.0)$ \\
\hline Chest pain & $12(16.0)$ \\
\hline Asymptomatic & $4(5.3)$ \\
\hline Surgical history & \\
\hline Nissen fundoplication & 10 \\
\hline Roux-en-Y gastric bypass & 1 \\
\hline Sleeve gastrectomy & 3 \\
\hline Subtotal gastrectomy & 1 \\
\hline Hiatal hernia (\%) & $10(13.3)$ \\
\hline Narcotic prescription (\%) & $21(28.0)$ \\
\hline Esophagram (\%) & 57 \\
\hline Dysmotility & $25(43.8)$ \\
\hline Stasis & $13(22.8)$ \\
\hline Tapering at GEJ & $5(8.8)$ \\
\hline EGD findings (\%) & 61 \\
\hline Dilated esophagus & $3(4.9)$ \\
\hline Tortuous esophagus & $3(4.9)$ \\
\hline Esophagitis & $3(4.9)$ \\
\hline
\end{tabular}

TABLE 2 Comparison of idiopathic and structural EGJOO

\begin{tabular}{|c|c|c|}
\hline & Idiopathic (N=65) & Structural $(\mathrm{N}=10)^{\mathrm{b}}$ \\
\hline $\begin{array}{l}\text { Mean CBT frequency } \\
\text { [SD] }\end{array}$ & $0.86[0.24]$ & 0.82 [0.33] \\
\hline $\begin{array}{l}\text { Mean FC frequency } \\
\text { [SD] }\end{array}$ & $0.81[0.30]$ & $0.66[0.46]$ \\
\hline $\begin{array}{l}\text { Median DCl } \\
(\mathrm{mmHg}-\mathrm{s}-\mathrm{cm})[\mathrm{IQR}]^{\mathrm{a}}\end{array}$ & 1992[1054-314] & $4384[976-5221]$ \\
\hline $\begin{array}{l}\text { Median IRP (mm Hg) } \\
\text { [IQR] }\end{array}$ & $18.6[16.8-22.6]$ & $18.8[17.1-20.9]$ \\
\hline \multicolumn{3}{|c|}{ 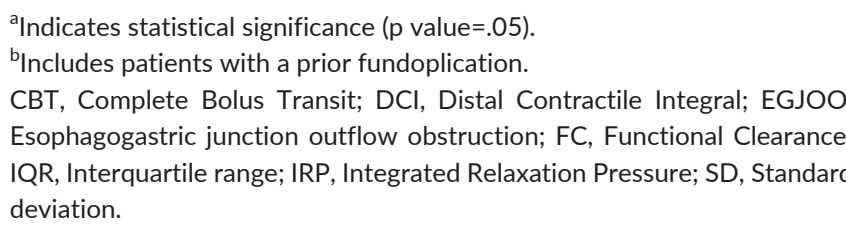 } \\
\hline
\end{tabular}

\section{3 | Comparison of idiopathic and structural EGJOO}

Presence of dysphagia, IRP and $\mathrm{DCl}$, and clearance measures per patient for idiopathic and structural EGJOO were compared. Dysphagia was more prevalent in the structural EGJOO group, $90 \%$ vs $64.6 \%(P=.04)$. HRIM data from the two groups is displayed in Table 2. In supine swallows, IRP was not significantly different between the two groups; however, $\mathrm{DCl}$ was higher in the structural 


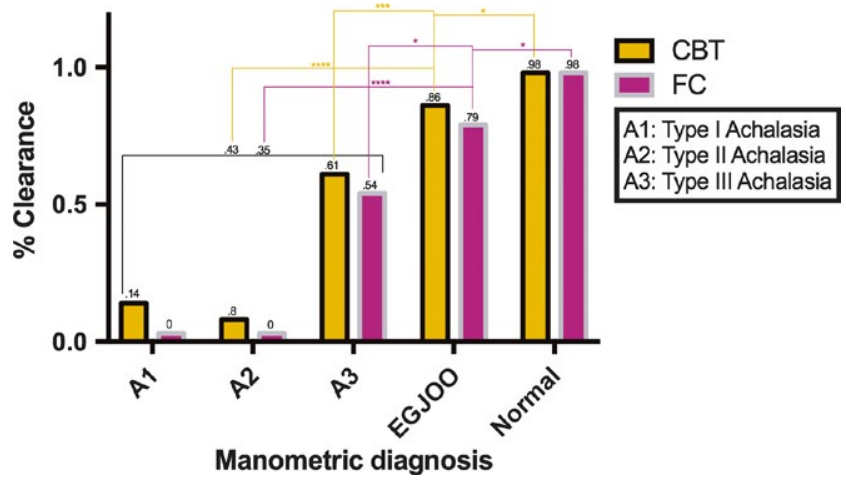

FIGURE 2 Bolus clearance by manometric diagnosis. Mean clearance is depicted as measured by Complete Bolus Transit (CBT) and Functional Clearance $(\mathrm{FC})$ in esophagogastric junction outflow obstruction (EGJOO) compared to the different achalasia subtypes, achalasia as a whole, and normal patients. Lines indicate comparisons between groups. Asterisks $\left({ }^{*}\right)$ indicate magnitude of statistical significance.

EGJOO group, median 4384 vs 1992 mmHg-s-cm ( $P=.05)$. There were no differences in CBT or FC between the groups in supine and upright swallows.

We also compared patients with EGJOO in the setting of any prior upper gastrointestinal (UGI) tract surgery and patients with EGJOO in the setting of narcotics separately to patients with other EGJOO. Results are displayed in Table 3. No significant differences were found in $\mathrm{CBT}, \mathrm{FC}, \mathrm{DCl}$, or IRP between the other EGJOO group when compared to the UGI tract surgery group or narcotic-prescribed groups.

\section{4 | Integrated relaxation pressure and distal contractile integral in association with complete bolus transit}

ROC curves in idiopathic EGJOO for CBT $\geq 60 \%$ and $\geq 80 \%$ per patient, and CBT clearance cumulatively across supine swallows in association with $\mathrm{DCl}$ and IRP are depicted in Figure 3. Areas-under-the-curve (AUCs) for IRP were 0.62, 0.52, and 0.60, respectively. These AUCs indicate a poor discriminatory capability of IRP for CBT. Thus, further threshold point analysis was not performed.

AUCs for $\mathrm{DCl}$ were $0.86,0.89$, and 0.87 , respectively. Cutoff points were chosen at a mean $\mathrm{DCl} 610 \mathrm{mmHg}$-s-cm for CBT $\geq 60 \%$, mean DCl 1513 mmHg-s-cm for CBT $\geq 80 \%$, and DCl $884 \mathrm{mmHg}$-s-cm for CBT clearance per swallow. A mean $\mathrm{DCl}>610 \mathrm{mmHg}-\mathrm{s}-\mathrm{cm}$ had an accuracy of $87.7 \%$ and a positive predictive value of $96.4 \%$ for bolus clearance as defined as CBT $\geq 60 \%(P=.004)$. A mean DCl $>1513 \mathrm{mmHg}$-s-cm had an accuracy of $76.9 \%$ and a positive predictive value of $97.5 \%$ for bolus clearance defined as $C B T \geq 80 \%$ ( $P<.0001)$. A $\mathrm{DCl}>884 \mathrm{mmHg}-\mathrm{s}-\mathrm{cm}$ had an accuracy of $81.9 \%$ and a positive predictive value of $97.8 \%$ for complete bolus transit for each individual swallow $(P<.0001)$.

\section{5 | Upright clearance}

We analyzed upright clearance in the 57 idiopathic EGJOO patients with available upright swallows. Kappa coefficient between CBT and FC frequency for clearance in $\geq 4$ of 5 swallows was good at 0.78 . We also constructed an ROC curve by mean $\mathrm{DCl}$ for clearance defined as CBT in all 5 upright swallows per patient. The AUC was 0.73. A mean $\mathrm{DCl}$ of $736 \mathrm{mmHg}-\mathrm{s}-\mathrm{cm}$ was chosen as the cutoff point, which had an accuracy of $73.7 \%$ for $C B T$ in all upright swallows ( $P=.0007)$. A mean $\mathrm{DCl}$ of $<736 \mathrm{mmHg}-\mathrm{s}-\mathrm{cm}$ was associated with incomplete upright bolus transit in $92.3 \%$ of patients.

Of note, two patients in our idiopathic EGJOO group had a DCl $>8000 \mathrm{mmHg}-\mathrm{s}-\mathrm{cm}$ on two or more swallows. Both patients had complete bolus transit on supine and upright swallows.

\subsection{Outcome measures}

Subject weight for at least 3 months prior to HRIM was available in 54 patients (72\%). Median weight change per patient as extrapolated to 1 year in all EGJOO patients was -2.5 [IQR $=-9$ to 3$]$ pounds. Clearance, DCl and IRP did not correlate with weight loss. Patient reported outcome questionnaires were collected in only a small number of patients at the time of HRIM. Median Eckardt score in 27 patients was 3 [IQR 1-4]. Median Impaction Dysphagia Questionnaire (IDQ) score in 22 patients was 7 [IQR 0-15.25]. We found no significant correlation between Eckardt or IDQ scores and clearance, but we were underpowered to assess this outcome.

\section{4 | DISCUSSION}

EGJOO was recently included as a motility disorder in the Chicago Classification. Its etiopathogenesis and long-term evolution still

TABLE 3 Comparison of EGJOO subgroups

\begin{tabular}{llll} 
& UGI tract surgery $(\mathrm{N}=15)^{\mathrm{a}}$ & Narcotic-prescribed $(\mathrm{N}=13)$ & Other EGJOO (N=47) \\
\hline Mean CBT frequency [SD] & $0.85[0.28]$ & $0.89[0.27]$ & $0.85[0.25]$ \\
\hline Mean FC frequency [SD] & $0.71[0.40]$ & $0.85[0.28]$ & $0.79[0.32]$ \\
\hline Median DCI (mmHg-s-cm) [IQR] & $2487[1030-4699]$ & $1837[1133-3281]$ & $1992[934-3200]$ \\
\hline Median IRP (mmHg) [IQR] & $19.1[17.3-22.0]$ & $18.0[17.3-22.0]$ & $18.6[16.8-22.8]$
\end{tabular}

ancludes patients with any esophagogastric surgery.

CBT, Complete Bolus Transit; DC, Distal Contractile Integral; EGJOO, Esophagogastric junction outflow obstruction; FC, Functional Clearance; IQR, Interquartile range; IRP, Integrated Relaxation Pressure; SD, Standard deviation. 


\section{(A) DCl and IRP with CBT across individual swallows}

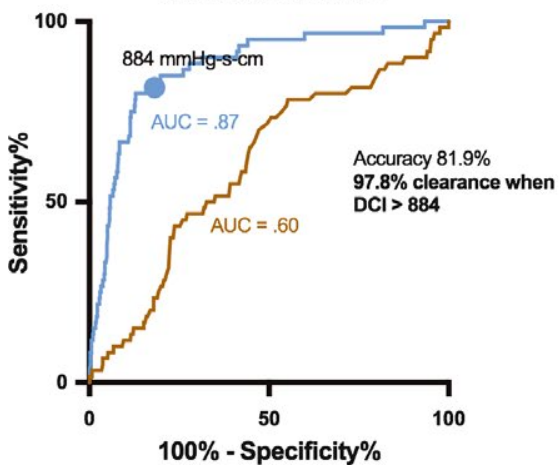

Association of $\mathrm{DCl}$ and IRP with Complete Bolus Transit

(B)

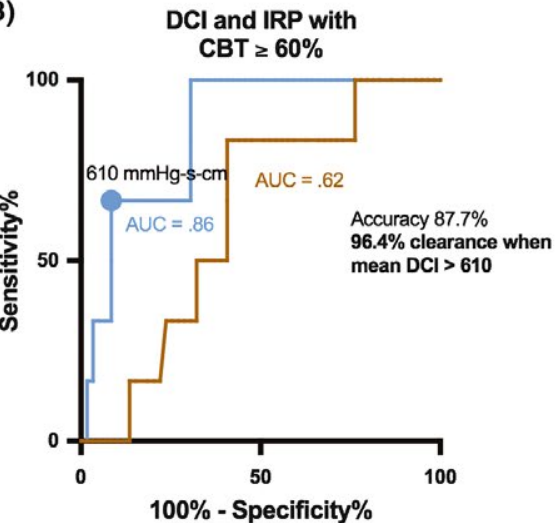

(C)

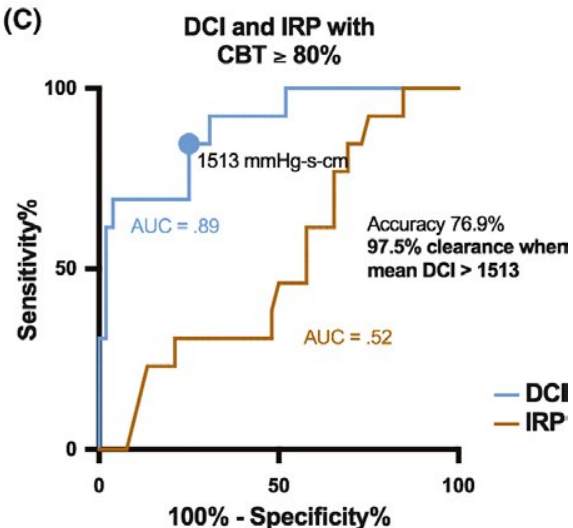

FIGURE 3 Receiver Operating Characteristic (ROC) curves depicting the association of Integrated Relaxation Pressure (IRP) and Distal Contractile Integral (DCI) with Complete Bolus Transit (CBT) at multiple thresholds across supine swallows. Area-under-the-curve (AUC) shown at each threshold. For DCl curves, accuracy and positive predictive value shown for cutoff points labeled on graphs.

remain to be fully understood. In the meantime, a manometric diagnosis of EGJOO in the absence of mechanical obstruction remains a management conundrum, in part since its clinical significance is uncertain. The aim of this study was to define functional phenotypes of idiopathic EGJOO based on bolus transit as assessed on intraluminal impedance. As we hypothesized, there is an overall impairment in bolus transit in EGJOO compared to the normal population, however not to the degree seen in achalasia. In comparing idiopathic to structural EGJOO, we note a decreased $\mathrm{DCl}$. This suggests a lack of compensatory hypercontraction to overcome a distal obstruction in EGJOO and implies a disorder in primary peristalsis. This bolsters the theory of idiopathic EGJOO as an achalasia spectrum disorder.

Our study adds to the current understanding of the clinical relevance of idiopathic EGJOO. Prior studies have mainly provided observational data regarding the symptoms, esophageal testing, and short-term clinical outcome ${ }^{4,7}$ of EGJOO patients. A recent study comparing bolus emptying on timed barium esophagram in structural vs idiopathic EGJOO patients found no difference between radiographic metrics. ${ }^{5}$ To our knowledge, a dedicated assessment of bolus transit has not been performed in idiopathic EGJOO to date. Our analysis included the largest cohort of idiopathic EGJOO patients to date and utilized a validated measure of bolus transit on impedance (CBT). ${ }^{9}$

Our key finding is that a DCl of around $900 \mathrm{mmHg}-\mathrm{s}-\mathrm{cm}$ or greater is associated with bolus clearance in idiopathic EGJOO in $97.8 \%$ of swallows. This threshold may identify patients with an adequate distal esophageal peristalsis to overcome the outflow obstruction. We would thus propose that patients who have had a mechanical obstruction excluded and have a DCl above this threshold are unlikely to have a significant functional esophageal disorder and can be observed clinically. Patients with a lower $\mathrm{DCl}$ could justifiably undergo therapy directed at the EGJ depending on the degree of symptoms and impairment in clearance. Notably, the degree of IRP elevation was not associated with bolus clearance. However, future studies using impedance planimetry to measure EGJ distensibility may further elucidate the flow dynamics across the EGJ in this patient population.
We observed a great deal of heterogeneity within EGJOO patients in terms of symptoms and severity, and some had other findings including hiatal hernias (rare in achalasia). We did not classify patients with EGJOO in the setting of gastric surgery separate from the esophagogastric junction as mechanical EGJOO. However, it is possible that these patients have some degree of obstruction below the EGJ that could impair clearance. In the literature available on this topic, migrated laparoscopic adjustable gastric bands are the only potential cause of EGJOO. Sleeve gastrectomy has been associated with both a decrease and an increase in LES tone, and RNYGB has minimal effect on motility ${ }^{14}$ Esophageal clearance has not been directly studied in this group of patients. Additionally, a non-insignificant portion of our cohort had a narcotic prescription, which has been associated with EGJOO finding on manometry previously. ${ }^{15}$ We found no significant functional differences between narcotic-related and otherwise idiopathic EGJOO in our cohort. Identifying patients with idiopathic EGJOO that will progress to achalasia remains a challenge. Prior cohorts suggest that many idiopathic EGJOO resolve spontaneously. Newer data also suggest that the presence of spastic motor patterns in EGJOO may denote evolving achalasia. ${ }^{16}$ The loss of peristaltic reserve as detected using multiple rapid swallows may be helpful in identification of these patients. ${ }^{17}$ This was not assessed in our current study.

HRIM offers a significant practical advantage over timed barium esophagram or videofluoroscopy, but the lack of an easily acquired and accurate measure of bolus transit has limited its adoption for this purpose in clinical practice. Complex metrics such as bolus flow time require sophisticated software algorithms, limiting their widespread use. ${ }^{18}$ Our study showed good agreement between an established and objective line-tracing method of assessing bolus transit and the more convenient contour method on a standard supine HRIM protocol as well as in upright swallows. This supports the use of these parameters as clinical indicators of functional bolus transit.

Our study has several limitations. Chief among them is the lack of correlation of bolus clearance or other manometric parameters with clinical outcomes. Bolus clearance did not correlate with weight loss in our cohort, and we were under-powered to analyze our outcome data 
at this point. Ongoing patient reported outcome data collection will enable future analysis on clinical outcomes in the EGJOO population. Our study is also subject to the inherent limitations of a retrospective study including a concern for analysis bias during unblinded interpretation of HRIM. However, objective criteria for analysis of bolus clearance were employed to limit this bias, and a second interpreter was able to reproduce the findings in a subset of data with a high degree of agreement. Despite prior validation of impedance as an accurate measure of bolus transit in multiple patient populations, the protocols used for prior studies were not directly transferable to the HRIM EPT analysis used in our study. Additionally, the use of multiple CBT thresholds was included in our analysis due to the uncertainty regarding the clinically meaningful threshold. Yet, the exact threshold used led to only minor differences in our results. Further study correlating CBT and FC with videofluoroscopy in supine and upright positions would be beneficial.

In summary, our study shows that patients with EGJOO have impaired bolus clearance compared to normal subjects but not to the severity seen in achalasia. In idiopathic EGJOO, weak peristalsis is associated with poor bolus clearance. In patients with a $\mathrm{DCl}$ greater than $900 \mathrm{mmHg}-\mathrm{s}-\mathrm{cm}$, a manometric finding of EGJOO is unlikely to be associated with impaired esophageal clearance. Future studies are needed to correlate objective disease measures with symptom-based and long-term outcomes in this patient population. Studies involving patient reported outcomes in correlation with HRM, impedance, and distensibility parameters may help develop diagnostic and therapeutic strategies for this complex population.

\section{DISCLOSURE}

No competing interests.

\section{AUTHOR CONTRIBUTION}

AJ Study planning, collection and analysis of data, interpretation of the data, and drafting of manuscript; JB collection of data, interpretation of the data, and editing the manuscript; JR Study planning, interpretation of the data, and editing the final manuscript; JC study planning, interpretation of data, editing and approval of final manuscript.

\section{REFERENCES}

1. Carlson DA, Pandolfino JE. The Chicago criteria for esophageal motility disorders: what has changed in the past 5 years? Curr Opin Gastroenterol. 2012;28:395-402.

2. Kahrilas PJ, Peters JH. Evaluation of the esophagogastric junction using high resolution manometry and esophageal pressure topography. Neurogastroenterol Motil. 2012;24(Suppl 1):11-19.

3. Kahrilas PJ, Bredenoord AJ, Fox M, et al. The Chicago Classification of esophageal motility disorders, v3.0. Neurogastroenterol Motil. 2015;27:160-174.
4. van Hoeij FB, Smout AJ, Bredenoord AJ. Characterization of idiopathic esophagogastric junction outflow obstruction. Neurogastroenterol Motil. 2015;27:1310-1316.

5. Clayton SB, Patel R, Richter JE. Functional and anatomic esophagogastic junction outflow obstruction: manometry, timed barium esophagram findings, and treatment outcomes. Clin Gastroenterol Hepatol. 2016;14:907-911.

6. DeLay K, Austin GL, Menard-Katcher P. Anatomic abnormalities are common potential explanations of manometric esophagogastric junction outflow obstruction. Neurogastroenterol Motil. 2016;28: 1166-1171.

7. Perez-Fernandez MT, Santander C, Marinero A, Burgos-Santamaria D, Chavarria-Herbozo C. Characterization and follow-up of esophagogastric junction outflow obstruction detected by high resolution manometry. Neurogastroenterol Motil. 2016;28:116-126.

8. Tutuian R, Vela MF, Balaji NS, et al. Esophageal function testing with combined multichannel intraluminal impedance and manometry: multicenter study in healthy volunteers. Clin Gastroenterol Hepatol. 2003;1:174-182.

9. Bogte A, Bredenoord AJ, Oors J, Siersema PD, Smout AJ. Assessment of bolus transit with intraluminal impedance measurement in patients with esophageal motility disorders. Neurogastroenterol Motil. 2015;27:1446-1452.

10. Cho YK, Choi MG, Lim CH, et al. Impaired esophageal bolus transit in patients with gastroesophageal reflux disease and abnormal esophageal Acid exposure. Gut Liver. 2012;6:440-445.

11. Omari TI, Szczesniak MM, Maclean J, et al. Correlation of esophageal pressure-flow analysis findings with bolus transit patterns on videofluoroscopy. Dis Esophagus. 2016;29:166-173.

12. Cho YK, Lipowska AM, Nicodeme F, et al. Assessing bolus retention in achalasia using high-resolution manometry with impedance: a comparator study with timed barium esophagram. Am J Gastroenterol. 2014;109:829-835.

13. Park MI. Recent concept in interpreting high-resolution manometry. J Neurogastroenterol Motil. 2010;16:90-93.

14. Naik RD, Choksi YA, Vaezi MF. Impact of weight loss surgery on esophageal physiology. Gastroenterol Hepatol (NY). 2015;11:801-809.

15. Ratuapli SK, Crowell MD, DiBaise JK, et al. Opioid-induced esophageal dysfunction (OIED) in patients on chronic opioids. Am J Gastroenterol. 2015;110:979-984.

16. Carlson DA, Lin Z, Kahrilas PJ, et al. The functional lumen imaging probe detects esophageal contractility not observed with manometry in patients with achalasia. Gastroenterology. 2015;149:1742-1751.

17. Shaker A, Stoikes N, Drapekin J, Kushnir V, Brunt LM, Gyawali CP. Multiple rapid swallow responses during esophageal high-resolution manometry reflect esophageal body peristaltic reserve. Am J Gastroenterol. 2013;108:1706-1712.

18. Carlson DA, Omari T, Lin Z, et al. High-resolution impedance manometry parameters enhance the esophageal motility evaluation in nonobstructive dysphagia patients without a major Chicago Classification motility disorder. Neurogastroenterol Motil. 2016;29(3). https://doi. org/20.2222/nmo.12941.

How to cite this article: Jain A, Baker JR, Rubenstein JH, Chen JW. Bolus clearance in esophagogastric junction outflow obstruction is associated with strength of peristalsis. Neurogastroenterol Motil. 2017;29:e13093. https://doi.org/ 10.1111/nmo.13093 